\title{
Rate of convergence for generalized Szász operators
}

\author{
Tuncer Acar • Vijay Gupta • Ali Aral
}

Received: 27 December 2010 / Revised: 26 February 2011 / Accepted: 8 March 2011 / Published online: 25 March 2011

(C) The Author(s) 2011. This article is published with open access at SpringerLink.com

\begin{abstract}
The present papers deals with the general integral modification of the Szász -Mirakyan operators having the weight functions of Baskakov basis functions. Here we estimate the rate of convergence for functions having derivatives of bounded variation.
\end{abstract}

Keywords Bounded variation · Szász-Mirakyan operators ·

Baskakov basis functions · Simultantaneous approximation · Q analogue

Mathematics Subject Classification (2000) Primary 41A25 · 41A35

Communicated by A. Laptev.

The work was done jointly, while V. Gupta visited Kırıkkale University during September 2010, supported by The Scientific and Technological Research Council of Turkey.

T. Acar $(\varangle) \cdot$ A. Aral

Department of Mathematics, Faculty of Science and Arts,

Yahşihan, Kirikkale, Turkey

e-mail: tunceracar@kku.edu.tr

A. Aral

e-mail: aliara173@yahoo.com

Present Address:

V. Gupta

School of Applied Sciences, Netaji Subhas Institute of Technology,

Sector 3 Dwarka, New Delhi 110078, India

e-mail: vijaygupta2001@hotmail.com 


\section{Introduction}

In the year 1983, Prasad et al [3] considered the integral modification of the well known Szász-Mirakyan operators by taking the weight functions of Baskakov operators and established some direct results in ordinary and simultaneous approximation. It was observed that there are some mistakes in [3], which were later improved by Gupta [1]. The general integral modification of the Szász-Mirakyan operators to approximate Lebesgue integrable functions on the interval $[0, \infty)$, with weight functions of Baskakov basis functions can be defined as

$$
G_{n, r}(f, x)=\frac{n^{r}(n-r-1) !}{(n-2) !} \sum_{k=0}^{\infty} s_{n, k}(x) \int_{0}^{\infty} b_{n-r, k+r}(t) f(t) d t, \quad r \geq 0
$$

where $n \in N, r \in N^{0}, n>r$ and the Szász and Baskakov basis functions are defined as

$$
s_{n, k}(x)=\frac{e^{-n x}(n x)^{k}}{k !}, \quad b_{n, k}(t)=\left(\begin{array}{c}
n+k-1 \\
k
\end{array}\right) \frac{t^{k}}{(1+t)^{n+k}} .
$$

The family of operators $G_{n, r}(f, x)$ is linear and positive. In the recent years the rate of convergence for functions of bounded variation is an active area of research, several researchers have studied in this direction, we refer some of the important papers in this area as [4-6] and [7] etc. Here we extent the studies and in the present paper, we estimate the rate of convergence for functions having derivatives of bounded variation. We have also studied the simultaneous approximation and in the end we mentioned an open problem for the readers.

\section{Auxiliary results}

Lemma 1 Let the mth order moment be defined as

$$
T_{n, r, m}(x)=(n-r-1) \sum_{k=0}^{\infty} s_{n, k}(x) \int_{0}^{\infty} b_{n-r, k+r}(t)(t-x)^{m} d t
$$

then for $n>r+m+2$, we have the following recurrence relation

$$
\begin{aligned}
(n-m-r-2) T_{n, r, m+1}(x)=x & {\left[T_{n, r, m}^{\prime}(x)+m(2+x) T_{n, r, m-1}(x)\right] } \\
+ & {[(m+r+1)+x(2 m+r+2)] T_{n, r, m}(x) . }
\end{aligned}
$$


Also

$$
\begin{aligned}
& T_{n, r, 0}(x)=1 \\
& T_{n, r, 1}(x)=\frac{(1+r)+x(r+2)}{n-r-2} \\
& T_{n, r, 2}(x)=\frac{x^{2}\left(n+5 r+r^{2}+6\right)+x\left(2 n+2 r^{2}+8 r+6\right)+r^{2}+3 r+2}{(n-r-3)(n-r-2)} .
\end{aligned}
$$

For all $r, m \in N_{0}$ and $x \in[0, \infty)$, one can easily obtain from the recurrence formula that

$$
T_{n, r, m}(x)=O_{x}\left(n^{-[(m+1) / 2]}\right)
$$

Proof Using the identities

$$
x s_{n, k}^{\prime}(x)=(k-n x) s_{n, k}(x)
$$

and

$$
t(1+t) b_{n-r, k+r}^{\prime}(t)=[(k+r)-(n-r) t] b_{n-r, k+r}(t)
$$

we have

$$
\begin{aligned}
& x T_{n, r, m}^{\prime}(x)=(n-r-1) \sum_{k=0}^{\infty} x s_{n, k}^{\prime}(x) \int_{0}^{\infty} b_{n-r, k+r}(t)(t-x)^{m} d t \\
& -x m(n-r-1) \sum_{k=0}^{\infty} s_{n, k}(x) \int_{0}^{\infty} b_{n-r, k+r}(t)(t-x)^{m-1} d t \\
& =(n-r-1) \sum_{k=0}^{\infty}(k-n x) s_{n, k}(x) \int_{0}^{\infty} b_{n-r, k+r}(t)(t-x)^{m} d t-m x T_{n, r, m-1}(x) \\
& x\left[T_{n, r, m}^{\prime}(x)+m T_{n, r, m-1}(x)\right] \\
& =(n-r-1) \sum_{k=0}^{\infty} s_{n, k}(x) \int_{0}^{\infty}(k-n x) b_{n-r, k+r}(t)(t-x)^{m} d t \\
& =(n-r-1) \sum_{k=0}^{\infty} s_{n, k}(x) \\
& \times \int_{0}^{\infty}[((k+r)-(n-r) t)+(n-r)(t-x)-r(1+x)] b_{n-r, k+r}(t)(t-x)^{m} d t \\
& =(n-r-1) \sum_{k=0}^{\infty} s_{n, k}(x) \int_{0}^{\infty} t(1+t) b_{n-r, k+r}^{\prime}(t)(t-x)^{m} d t
\end{aligned}
$$




$$
\begin{aligned}
& +(n-r)(n-r-1) \sum_{k=0}^{\infty} s_{n, k}(x) \int_{0}^{\infty} b_{n-r, k+r}(t)(t-x)^{m+1} d t \\
& -r(1+x)(n-r-1) \sum_{k=0}^{\infty} s_{n, k}(x) \int_{0}^{\infty} b_{n-r, k+r}(t)(t-x)^{m} d t \\
& =(n-r-1) \sum_{k=0}^{\infty} s_{n, k}(x) \\
& \quad \times \int_{0}^{\infty} b_{n-r, k+r}^{\prime}(t)\left[(t-x)^{m+2}+(1+2 x)(t-x)^{m+1}+x(1+x)(t-x)^{m}\right] d t \\
& +(n-r) T_{n, r, m+1}(x)-r(1+x) T_{n, r, m}(x)
\end{aligned}
$$

Now integrating by parts the last integral, we get

$$
\begin{aligned}
& x\left[T_{n, r, m}^{\prime}(x)+m T_{n, r, m-1}(x)\right] \\
& =-(m+2) T_{n, r, m+1}(x)-(m+1)(1+2 x) T_{n, r, m}(x) \\
& \quad-x(1+x) m T_{n, r, m-1}(x)+(n-r) T_{n, r, m+1}(x)-r(1+x) T_{n, r, m}(x)
\end{aligned}
$$

Thus

$$
\begin{aligned}
& (n-r-m-2) T_{n, r, m+1}(x)=x\left[T_{n, r, m}^{\prime}(x)+m(2+x) T_{n, r, m-1}(x)\right] \\
& \quad+(m+1)(1+2 x) T_{n, r, m}(x)+r(1+x) T_{n, r, m}(x) .
\end{aligned}
$$

The moments can be obtained easily by using the above recurrence relation, keeping in mind the fact that $\sum_{k=0}^{\infty} s_{n, k}(x)=1$ and $\int_{0}^{\infty} b_{n, k}(t) d t=\frac{1}{n-1}$, we omit the details.

Remark 1 For $n$ sufficiently large, $C>1$ and $x \in(0, \infty)$, it can be seen from Lemma 1 , that

$$
\frac{x(x+2)}{n-r-3} \leq T_{n, r, 2}(x) \leq \frac{C\left(x^{2}+2 x\right)}{n-r-3}
$$

Remark 2 By using Cauchy-Schwarz inequality, it follows from Remark 1, that for $n$ sufficiently large , $C>1$ and $x \in(0, \infty)$

$$
(n-r-1) \sum_{k=0}^{\infty} s_{n, k}(x) \int_{0}^{\infty} b_{n-r, k+r}(t)|t-x| d t \leq\left[T_{n, r, 2}(x)\right]^{\frac{1}{2}} \leq \sqrt{\frac{C x(x+2)}{n-r-3}}
$$


Lemma 2 Let $x \in(0, \infty)$ and $C>1$, then for $n$ sufficiently large, we have

$$
\begin{aligned}
& \lambda_{n, r}(x, y)=(n-r-1) \sum_{k=0}^{\infty} s_{n, k}(x) \int_{0}^{y} b_{n-r, k+r}(t) d t \leq \frac{C x(x+2)}{(n-r-3)(x-y)^{2}}, \\
& 0 \leq y<x \\
& 1-\lambda_{n, r}(x, z)=(n-r-1) \sum_{k=0}^{\infty} s_{n, k}(x) \int_{z}^{\infty} b_{n-r, k+r}(t) d t \leq \frac{C x(x+2)}{(n-r-3)(z-x)^{2}}, \\
& x<z<\infty .
\end{aligned}
$$

Proof The proof of the above lemma follows easily by using Remark 1. For instance, for the first inequality for $n$ sufficiently large and $0 \leq y<x$, we have

$$
\begin{aligned}
\lambda_{n, r}(x, y) & =(n-r-1) \sum_{k=0}^{\infty} s_{n, k}(x) \int_{0}^{y} b_{n-r, k+r}(t) d t \\
& \leq(n-r-1) \sum_{k=0}^{\infty} s_{n, k}(x) \int_{0}^{y} b_{n-r, k+r}(t) \frac{(t-x)^{2}}{(y-x)^{2}} d t \\
& =\frac{T_{n, r, 2}(x)}{(y-x)^{2}} \leq \frac{C x(x+2)}{(n-r-3)(x-y)^{2}} .
\end{aligned}
$$

The proof of the second inequality follows along the similar lines.

Lemma 3 Suppose $f$ is s times differentiable on $[0, \infty)$ such that $f^{(s-1)}(t)=O\left(t^{q}\right)$, for some positive integer $q$ as $t \rightarrow \infty$. Then for any $r, s \in N_{0}$ and $n>\max \{q, r+$ $s+1\}$, we have

$$
D^{s} G_{n, r}(f, x)=G_{n, r+s}\left(D^{s} f, x\right)
$$

Proof Using the identities

$$
\begin{gathered}
D s_{n, k}(x)=n\left[s_{n, k-1}(x)-s_{n, k}(x)\right] \\
D b_{n, k}(t)=n\left[b_{n+1, k-1}(t)-b_{n+1, k}(t)\right]
\end{gathered}
$$

One can observe that even in case $k=0$, the above identities are true with the conditions that $b_{n+1, \text { negative }}(x)=0$ and $s_{n, \text { negative }}(x)=0$, thus applying (2.3), we 
have

$$
\begin{aligned}
D G_{n, r}(f, x) & =\frac{n^{r}(n-r-1) !}{(n-2) !} \sum_{k=0}^{\infty} D s_{n, k}(x) \int_{0}^{\infty} b_{n-r, k+r}(t) f(t) d t \\
& =\frac{n^{r+1}(n-r-1) !}{(n-2) !} \sum_{k=0}^{\infty}\left[s_{n, k-1}(x)-s_{n, k}(x)\right] \int_{0}^{\infty} b_{n-r, k+r}(t) f(t) d t \\
& =\frac{n^{r+1}(n-r-1) !}{(n-2) !} \sum_{k=0}^{\infty} s_{n, k}(x) \int_{0}^{\infty}\left[b_{n-r, k+r+1}(t)-b_{n-r, k+r}(t)\right] f(t) d t .
\end{aligned}
$$

Using (2.4), and integrating by parts we have

$$
\begin{aligned}
D G_{n, r}(f, x) & =\frac{n^{r+1}(n-r-1) !}{(n-2) !} \sum_{k=0}^{\infty} s_{n, k}(x) \int_{0}^{\infty}-\frac{D\left[b_{n-r-1, k+r+1}(t)\right]}{n-r-1} f(t) d t \\
& =\frac{n^{r+1}(n-r-2) !}{(n-2) !} \sum_{k=0}^{\infty} s_{n, k}(x) \int_{0}^{\infty} b_{n-r-1, k+r+1}(t) f^{(1)}(t) d t=G_{n, r+1}(D f, x)
\end{aligned}
$$

which means that the identity is satisfied for $s=1$. Let us suppose that the result holds for $s$ i.e.,

$$
\begin{aligned}
D^{s} G_{n, r}(f, x)= & G_{n, r+s}\left(D^{s} f, x\right) \\
= & \frac{n^{r+s}(n-r-s-1) !}{(n-2) !} \sum_{k=0}^{\infty} s_{n, k}(x) \int_{0}^{\infty} b_{n-r-s, k+r+s}(t) D^{(s)} f(t) d t \\
D^{s+1} G_{n, r}(f, x)= & \frac{n^{r+s}(n-r-s-1) !}{(n-2) !} \sum_{k=0}^{\infty} D s_{n, k}(x) \\
& \times \int_{0}^{\infty} b_{n-r-s, k+r+s}(t) D^{s} f(t) d t \\
= & \frac{n^{r+s+1}(n-r-s-1) !}{(n-2) !} \sum_{k=0}^{\infty}\left[s_{n, k-1}(x)-s_{n, k}(x)\right] \\
& \times \int_{0}^{\infty} b_{n-r-s, k+r+s}(t) D^{s} f(t) d t
\end{aligned}
$$




$$
\begin{aligned}
= & \frac{n^{r+s+1}(n-r-s-1) !}{(n-2) !} \sum_{k=0}^{\infty} s_{n, k}(x) \\
& \times \int_{0}^{\infty}\left[b_{n-r-s, k+r+s+1}(t)-b_{n-r-s, k+r+s}(t)\right] D^{s} f(t) d t \\
= & \frac{n^{r+s+1}(n-r-s-1) !}{(n-2) !} \sum_{k=0}^{\infty} s_{n, k}(x) \\
& \times \int_{0}^{\infty}-\frac{D\left[b_{n-r-s-1, k+r+s+1}(t)\right]}{n-r-s-1} D^{s} f(t) d t .
\end{aligned}
$$

Integrating by parts the last integral we get

$$
\frac{n^{r+s+1}(n-r-s-2) !}{(n-2) !} \sum_{k=0}^{\infty} s_{n, k}(x) \int_{0}^{\infty} b_{n-r-s-1, k+r+s+1}(t) D^{s+1} f(t) d t .
$$

Thus we obtain that the result holds for $s+1$, hence by the mathematical induction the proof of the lemma is complete.

\section{Rate of convergence}

By $D B_{q}(0, \infty)$, (where $q$ is some positive integer) we mean the class of absolutely continuous functions $f$ defined on $(0, \infty)$ satisfying the following conditions:

(i) $f(t)=O\left(t^{q}\right), t \rightarrow \infty$

(ii) the function $f$ has the first derivative on the interval $(0, \infty)$ which coincide a.e. with a function which is of bounded variation on every finite subinterval of $(0, \infty)$. It can be observed that for all functions $f \in D B_{q}(0, \infty)$ we can have the representation

$$
f(x)=f(c)+\int_{c}^{x} \psi(t) d t, \quad x \geq c>0
$$

Theorem 1 Let $f \in D B_{q}(0, \infty), q>0$ and $x \in(0, \infty)$. Then for $C>1$ and $n$ sufficiently large, we have

$$
\begin{aligned}
& \left|\frac{(n-2) !}{n^{r}(n-r-2) !} G_{n, r}(f, x)-f(x)\right| \\
& \leq \frac{C(x+2)}{n-r-3}\left(\sum_{k=1}^{[\sqrt{n}] x+x / k} \bigvee_{x-x / k}\left(\left(f^{\prime}\right)_{x}\right)+\frac{x}{\sqrt{n}} \bigvee_{x-x / \sqrt{n}}^{x+x / \sqrt{n}}\left(\left(f^{\prime}\right)_{x}\right)\right)
\end{aligned}
$$




$$
\begin{aligned}
& +\frac{C(x+2)}{(n-r-3) x}\left(\left|f(2 x)-f(x)-x f^{\prime}\left(x^{+}\right)\right|+|f(x)|\right) \\
& +O\left(n^{-q}\right)+\left|f^{\prime}\left(x^{+}\right)\right| \frac{C(x+2)}{n-r-3}+\frac{1}{2} \sqrt{\frac{C x(x+2)}{n-r-3}}\left|f^{\prime}\left(x^{+}\right)-f^{\prime}\left(x^{-}\right)\right| \\
& +\frac{1}{2}\left|f^{\prime}\left(x^{+}\right)+f^{\prime}\left(x^{-}\right)\right| \frac{(2+r) x+(r+1)}{n-r-2},
\end{aligned}
$$

where $\bigvee_{a}^{b} f(x)$ denotes the total variation of $f_{x}$ on $[a, b]$, and $f_{x}$ is defined by

$$
f_{x}(t)= \begin{cases}f(t)-f\left(x^{-}\right), & 0 \leq t<x \\ 0, & t=x \\ f(t)-f\left(x^{+}\right), & x<t<\infty .\end{cases}
$$

Proof Using the mean value theorem, we can write

$$
\begin{aligned}
& \left|\frac{(n-2) !}{n^{r}(n-r-2) !} G_{n, r}(f, x)-f(x)\right| \\
& \leq(n-r-1) \sum_{k=0}^{\infty} s_{n, k}(x) \int_{0}^{\infty} b_{n-r, k+r}(t)|f(t)-f(x)| d t \\
& \quad=\int_{0}^{\infty}\left|\int_{x}^{t}(n-r-1) \sum_{k=0}^{\infty} s_{n, k}(x) b_{n-r, k+r}(t) f^{\prime}(u) d u\right| d t .
\end{aligned}
$$

Also, using the identity

$$
\begin{aligned}
f^{\prime}(u)= & \frac{f^{\prime}\left(x^{+}\right)+f^{\prime}\left(x^{-}\right)}{2}+\left(f^{\prime}\right)_{x}(u)+\frac{f^{\prime}\left(x^{+}\right)-f^{\prime}\left(x^{-}\right)}{2} \operatorname{sgn}(u-x) \\
& +\left[f^{\prime}(x)-\frac{f^{\prime}\left(x^{+}\right)+f^{\prime}\left(x^{-}\right)}{2}\right] \chi_{x}(u),
\end{aligned}
$$

where

$$
\chi_{x}(u)= \begin{cases}1, & u=x \\ 0, & u \neq x\end{cases}
$$

Obviously, we have

$$
\begin{aligned}
& (n-r-1) \int_{0}^{\infty}\left(\int_{x}^{t}\left(f^{\prime}(x)-\frac{f^{\prime}\left(x^{+}\right)+f^{\prime}\left(x^{-}\right)}{2}\right) \chi_{x}(u) d u\right) \\
& \times \sum_{k=0}^{\infty} s_{n, k}(x) b_{n-r, k+r}(t) d t=0 .
\end{aligned}
$$


Thus, using above identities, we can write

$$
\begin{aligned}
& \left|\frac{(n-2) !}{n^{r}(n-r-2) !} G_{n, r}(f, x)-f(x)\right| \\
& \leq\left|(n-r-1) \int_{0}^{\infty}\left(\int_{x}^{t} \sum_{k=0}^{\infty} s_{n, k}(x) b_{n-r, k+r}(t)\left(\frac{f^{\prime}\left(x^{+}\right)+f^{\prime}\left(x^{-}\right)}{2}+\left(f^{\prime}\right)_{x}(u)\right) d u\right) d t\right| \\
& \quad+\left|(n-r-1) \int_{0}^{\infty}\left(\int_{x}^{t} \sum_{k=0}^{\infty} s_{n, k}(x) b_{n-r, k+r}(t) \frac{\left[f^{\prime}\left(x^{+}\right)-f^{\prime}\left(x^{-}\right)\right]}{2} \operatorname{sgn}(u-x) d u\right) d t\right| .
\end{aligned}
$$

Also, it can be verified that

$$
\begin{gathered}
\left|(n-r-1) \int_{0}^{\infty}\left(\int_{x}^{t} \frac{\left[f^{\prime}\left(x^{+}\right)-f^{\prime}\left(x^{-}\right)\right]}{2} \operatorname{sgn}(u-x) d u\right) \sum_{k=0}^{\infty} s_{n, k}(x) b_{n-r, k+r}(t) d t\right| \\
\leq \frac{\left|f^{\prime}\left(x^{+}\right)-f^{\prime}\left(x^{-}\right)\right|_{\left[T_{n, r, 2}(x)\right]^{1 / 2}}}{2}
\end{gathered}
$$

and

$$
\begin{gathered}
(n-r-1) \int_{0}^{\infty}\left(\int_{x}^{t} \frac{\left[f^{\prime}\left(x^{+}\right)+f^{\prime}\left(x^{-}\right)\right]}{2} d u\right) \sum_{k=0}^{\infty} s_{n, k}(x) b_{n-r, k+r}(t) d t \\
=\frac{\left[f^{\prime}\left(x^{+}\right)+f^{\prime}\left(x^{-}\right)\right]}{2} T_{n, r, 1}(x) .
\end{gathered}
$$

Combining (3.1)-(3.3), we can have

$$
\begin{aligned}
& \left|\frac{(n-2) !}{n^{r}(n-r-2) !} G_{n, r}(f, x)-f(x)\right| \leq \mid(n-r-1) \int_{x}^{\infty}\left(\int_{x}^{t}\left(f^{\prime}\right)_{x}(u) d u\right) \\
& \quad \times \sum_{k=0}^{\infty} s_{n, k}(x) b_{n-r, k+r}(t) d t(n-r-1) \int_{0}^{x}\left(\int_{x}^{t}\left(f^{\prime}\right)_{x}(u) d u\right) \sum_{k=0}^{\infty} s_{n, k}(x) b_{n-r, k+r}(t) d t \mid \\
& \quad+\frac{\left|f^{\prime}\left(x^{+}\right)-f^{\prime}\left(x^{-}\right)\right|}{2}\left[T_{n, r, 2}(x)\right]^{1 / 2} \\
& \quad+\frac{\left|f^{\prime}\left(x^{+}\right)+f^{\prime}\left(x^{-}\right)\right|}{2} T_{n, r, 1}(x)
\end{aligned}
$$




$$
\begin{aligned}
= & \left|A_{n, r}(f, x)+B_{n, r}(f, x)\right|+\frac{\left|f^{\prime}\left(x^{+}\right)-f^{\prime}\left(x^{-}\right)\right|}{2}\left[T_{n, r, 2}(x)\right]^{1 / 2} \\
& +\frac{\left|f^{\prime}\left(x^{+}\right)+f^{\prime}\left(x^{-}\right)\right|}{2} T_{n, r, 1}(x) .
\end{aligned}
$$

Applying Remark 2 and Lemma 1, in (3.4), we have

$$
\begin{aligned}
& \left|\frac{(n-2) !}{n^{r}(n-r-2) !} G_{n, r}(f, x)-f(x)\right| \leq\left|A_{n, r}(f, x)\right|+\left|B_{n, r}(f, x)\right| \\
& \quad+\frac{\left|f^{\prime}\left(x^{+}\right)-f^{\prime}\left(x^{-}\right)\right|}{2} \sqrt{\frac{C\left(x^{2}+2 x\right)}{n-r-3}}+\frac{\left|f^{\prime}\left(x^{+}\right)+f^{\prime}\left(x^{-}\right)\right|}{2} \frac{(2+r) x+(r+1)}{n-r-2} .
\end{aligned}
$$

In order to complete the proof of the theorem it suffices to estimate the terms $A_{n, r}(f, x)$ and $B_{n, r}(f, x)$. Applying integration by parts and Lemma 2 with $y=x-x / \sqrt{n}$, we have

$$
\begin{aligned}
\left|B_{n, r}(f, x)\right| & =\left|\int_{0}^{x} \int_{x}^{t}\left(f^{\prime}\right)_{x}(u) d u d_{t}\left(\lambda_{n, r}(x, t)\right)\right| \\
& =\int_{0}^{x} \lambda_{n, r}(x, t)\left(f^{\prime}\right)_{x}(t) d t \leq\left(\int_{0}^{y}+\int_{y}^{x}\right)\left|\left(f^{\prime}\right)_{x}(t)\right|\left|\lambda_{n, r}(x, t)\right| d t \\
& \leq \frac{C x(x+2)}{n-r-3} \int_{0}^{y} \bigvee_{t}^{x}\left(\left(f^{\prime}\right)_{x}\right) \frac{1}{(x-t)^{2}} d t+\int_{y}^{x} \bigvee_{t}^{x}\left(\left(f^{\prime}\right)_{x}\right) d t \\
& \leq \frac{C x(x+2)}{n-r-3} \int_{0}^{y} \bigvee_{t}^{x}\left(\left(f^{\prime}\right)_{x}\right) \frac{1}{(x-t)^{2}} d t+\frac{x}{\sqrt{n}} \bigvee_{x-\frac{x}{\sqrt{n}}}^{x}\left(\left(f^{\prime}\right)_{x}\right) .
\end{aligned}
$$

Let $u=\frac{x}{x-t}$. Then we have

$$
\begin{aligned}
\frac{C x(x+2)}{n-r-3} \int_{0}^{y} \bigvee_{t}^{x}\left(\left(f^{\prime}\right)_{x}\right) \frac{1}{(x-t)^{2}} d t & =\frac{C x(x+2)}{n-r-3} \int_{1}^{\sqrt{n}} \bigvee_{x-\frac{x}{u}}^{x}\left(\left(f^{\prime}\right)_{x}\right) d u \\
& \leq \frac{C(x+2)}{n-r-3} \sum_{k=1}^{[\sqrt{n}]} \bigvee_{x-\frac{x}{k}}^{x}\left(\left(f^{\prime}\right)_{x}\right)
\end{aligned}
$$


Thus

$$
\left|B_{n, r}(f, x)\right| \leq \frac{C(x+2)}{n-r-3} \sum_{k=1}^{[\sqrt{n}]} \bigvee_{x-\frac{x}{k}}^{x}\left(\left(f^{\prime}\right)_{x}\right)+\frac{x}{\sqrt{n}} \bigvee_{x-\frac{x}{\sqrt{n}}}^{x}\left(\left(f^{\prime}\right)_{x}\right)
$$

On the other hand, we have

$$
\begin{aligned}
& \left|A_{n, r}(f, x)\right|=\left|(n-r-1) \int_{x}^{\infty}\left(\int_{x}^{t}\left(f^{\prime}\right)_{x}(u) d u\right) \sum_{k=0}^{\infty} s_{n, k}(x) b_{n-r, k+r}(t) d t\right| \\
& =\mid(n-r-1) \int_{2 x}^{\infty}\left(\int_{x}^{t}\left(f^{\prime}\right)_{x}(u) d u\right) \sum_{k=0}^{\infty} s_{n, k}(x) b_{n-r, k+r}(t) d t \\
& +\int_{x}^{2 x}\left(\int_{x}^{t}\left(f^{\prime}\right)_{x}(u) d u\right) d_{t}\left(1-\lambda_{n, r}(x, t)\right) \\
& \leq\left|(n-r-1) \sum_{k=0}^{\infty} s_{n, k}(x) \int_{2 x}^{\infty}(f(t)-f(x)) b_{n-r, k+r}(t) d t\right| \\
& +\left|f^{\prime}\left(x^{+}\right)\right|\left|(n-r-1) \sum_{k=0}^{\infty} s_{n, k}(x) \int_{2 x}^{\infty} b_{n-r, k+r}(t)(t-x) d t\right| \\
& +\left|\int_{x}^{2 x}\left(f^{\prime}\right)_{x}(u) d u\right| \mid\left(1-\lambda_{n, r}(x, 2 x)\left|+\int_{x}^{2 x}\right|\left(f^{\prime}\right)_{x}(t)||\left(1-\lambda_{n, r}(x, t) \mid d t\right.\right. \\
& \leq(n-r-1) \sum_{k=0}^{\infty} s_{n, k}(x) \int_{2 x}^{\infty} b_{n-r, k+r}(t) C_{1} t^{2 q} d t \\
& +\frac{|f(x)|}{x^{2}}(n-r-1) \sum_{k=0}^{\infty} s_{n, k}(x) \int_{2 x}^{\infty} b_{n-r, k+r}(t)(t-x)^{2} d t \\
& +\left|f^{\prime}\left(x^{+}\right)\right|(n-r-1) \int_{2 x}^{\infty} \sum_{k=0}^{\infty} s_{n, k}(x) b_{n-r, k+r}(t)|(t-x)| d t \\
& +\frac{C(x+2)}{(n-r-3) x}\left|f(2 x)-f(x)-x f^{\prime}\left(x^{+}\right)\right| \\
& +\frac{C(x+2)}{n-r-3} \sum_{k=1}^{[\sqrt{n}]} \bigvee_{x}^{x+\frac{x}{k}}\left(\left(f^{\prime}\right)_{x}\right)+\frac{x}{\sqrt{n}} \bigvee_{x}^{x+\frac{x}{\sqrt{n}}}\left(\left(f^{\prime}\right)_{x}\right) \text {. }
\end{aligned}
$$

To estimate the integral $(n-r-1) \sum_{k=0}^{\infty} s_{n, k}(x) \int_{2 x}^{\infty} b_{n-r, k+r}(t) C_{1} t^{2 q} d t$ in (3.7) above, we proceed as follows: 
Obviously $t \geq 2 x$ implies that $t \leq 2(t-x)$ and it follows from Lemma 1 , that

$$
\begin{aligned}
& (n-r-1) \sum_{k=0}^{\infty} s_{n, k}(x) \int_{2 x}^{\infty} b_{n-r, k+r}(t) t^{2 q} d t \leq C_{1} 2^{2 q}(n-r-1) \\
& \times \sum_{k=0}^{\infty} s_{n, k}(x) \int_{0}^{\infty} b_{n-r, k+r}(t)(t-x)^{2 q} d t=C_{1} 2^{2 q} T_{n, r, 2 q}(x)=O\left(n^{-q}\right)(n \rightarrow \infty) .
\end{aligned}
$$

Applying Schwarz inequality and Remark 1, third term in right hand side of (3.7) is estimated as follows:

$$
\begin{aligned}
& \left|f^{\prime}\left(x^{+}\right)\right|(n-r-1) \sum_{k=0}^{\infty} s_{n, k}(x) \int_{2 x}^{\infty} b_{n-r, k+r}(t)|t-x| d t \\
& \quad \leq \frac{\left|f^{\prime}\left(x^{+}\right)\right|}{x}(n-r-1) \sum_{k=0}^{\infty} s_{n, k}(x) \int_{0}^{\infty} b_{n-r, k+r}(t)(t-x)^{2} d t \\
& \quad=\left|f^{\prime}\left(x^{+}\right)\right| \frac{C(x+2)}{n-r-3} .
\end{aligned}
$$

Thus by Lemma 1 and Remark 1 , we have $\left|A_{n, r}(f, x)\right|$

$$
\begin{aligned}
\leq & \mathcal{O}\left(n^{-q}\right)+\left|f^{\prime}\left(x^{+}\right)\right| \cdot \frac{C(x+2)}{n-r-3} \\
& +\frac{C(x+2)}{(n-r-3) x}\left(\left|f(2 x)-f(x)-x f^{\prime}\left(x^{+}\right)\right|+|f(x)|\right) \\
& +\frac{C(x+2)}{n-r-3} \sum_{k=1}^{[\sqrt{n}]} \bigvee_{x}^{x+\frac{x}{k}}\left(\left(f^{\prime}\right)_{x}\right)+\frac{x}{\sqrt{n}} \bigvee_{x}^{x+\frac{x}{\sqrt{n}}}\left(\left(f^{\prime}\right)_{x}\right) .
\end{aligned}
$$

Collecting the estimates (3.5), (3.6) and (3.8), we get the required result. This completes the proof of Theorem 1 .

Using Lemma 3, we can easily prove the following result for the derivatives of the operators $G_{n, r}(f, x)$. 
Corollary 1 Let $f^{(s)} \in D B_{q}(0, \infty), q>0$ and $x \in(0, \infty)$. Then for $C>1$ and for $n$ sufficiently large, we have

$$
\begin{aligned}
& \left|\frac{(n-2) !}{n^{r}(n-r-2) !} D^{s} G_{n, r}(f, x)-f^{(s)}(x)\right| \leq \frac{C(x+2)}{n-r-3} \\
& \quad \times\left(\sum_{k=1}^{[\sqrt{n}] x+x / k}\left(\left(D^{s+1} f\right)_{x}\right)+\frac{x}{\sqrt{n}} \bigvee_{x-x / \sqrt{n}}^{x+x / \sqrt{n}}\left(\left(D^{s+1} f\right)_{x}\right)\right) \\
& +\frac{C(x+2)}{x(n-r-3)}\left(\left|D^{s} f(2 x)-D^{s} f(x)-x D^{s+1} f\left(x^{+}\right)\right|+\left|D^{s} f(x)\right|\right) \\
& +O\left(n^{-q}\right)+\frac{C(x+2)}{n-r-3}\left|D^{s+1} f\left(x^{+}\right)\right| \\
& +\frac{1}{2} \sqrt{\frac{C x(x+2)}{n}}\left|D^{s+1} f\left(x^{+}\right)-D^{s+1} f\left(x^{-}\right)\right| \\
& +\frac{1}{2}\left|D^{s+1} f\left(x^{+}\right)+D^{s+1} f\left(x^{-}\right)\right| \frac{(2+r) x+(r+1)}{n-r-2},
\end{aligned}
$$

where $\bigvee_{a}^{b} f(x)$ denotes the total variation of $f_{x}$ on $[a, b]$, and $f_{x}$ is defined by

$$
D^{s+1} f_{x}(t)= \begin{cases}D^{s+1} f(t)-D^{s+1} f\left(x^{-}\right), & 0 \leq t<x \\ 0, & t=x \\ D^{s+1} f(t)-D^{s+1} f\left(x^{+}\right), & x<t<\infty\end{cases}
$$

Remark 3 For every $n \in \mathbb{N}, q \in(0,1)$, the $q$ analogue of the operators (1.1) for the case $r=0$, can be defined as

$$
G_{n, 0}^{q}(f(t), x):=[n-1]_{q} \sum_{k=0}^{\infty} s_{n, k}^{q}(x) q^{k} \int_{0}^{\infty / A} b_{n, k}^{q}(t) f(t) d_{q} t
$$

where

$$
s_{n, k}^{q}(x)=\frac{\left([n]_{q} x\right)^{k}}{[k]_{q} !} q^{k(k-1) / 2} \frac{1}{E_{q}\left([n]_{q} x\right)}
$$

and

$$
b_{n, k}^{q}(t):=\left[\begin{array}{c}
n+k-1 \\
k
\end{array}\right]_{q} q^{k(k-1) / 2} \frac{t^{k}}{(1+t)_{q}^{n+k}}
$$

for $x \in[0, \infty)$ and for every real valued continuous function $f$ on $[0, \infty)$. We use the following notations of $q$ calculus (see [2] and references therein): 
For $n \in \mathbb{N}$, the $q$-integers, $q$-factorial and $q$-binomial coefficients are defined as

$$
\begin{gathered}
{[n]_{q}:=\frac{1-q^{n}}{1-q},} \\
{[n]_{q} !:= \begin{cases}{[n]_{q}[n-1]_{q} \cdots[1]_{q},} & n=1,2, \ldots \\
1, & n=0\end{cases} }
\end{gathered}
$$

and

$$
\left[\begin{array}{l}
n \\
k
\end{array}\right]_{q}=\frac{[n]_{q} !}{[k]_{q} ![n-k]_{q} !}
$$

The $q$ exponential function is defined as

$$
E_{q}(z)=\prod_{j=0}^{\infty}\left(1+(1-q) q^{j} z\right)=\sum_{k=0}^{\infty} q^{k(k-1) / 2} \frac{z^{k}}{[k]_{q} !}=(1+(1-q) z)_{q}^{\infty}, \quad|q|<1,
$$

where $(1+x)_{q}^{\infty}=\prod_{j=0}^{\infty}\left(1+q^{j} x\right)$ and $q$-improper integral is defined as

$$
\int_{0}^{\infty / A} f(x) d_{q} x=(1-q) \sum_{n=-\infty}^{\infty} f\left(\frac{q^{n}}{A}\right) \frac{q^{n}}{A}, \quad A>0
$$

provided the sums converge absolutely.

In [2], the authors have studied some other approximation properties on these $q$ operators, but we observe that the results analogous to the present paper are not possible for such $q$ operators even for special value i.e., $r=0$. This can be considered as an open problem.

Acknowledgments The authors are thankful to the referee for valuable suggestions leading to overall improvements in the paper.

Open Access This article is distributed under the terms of the Creative Commons Attribution License which permits any use, distribution and reproduction in any medium, provided the original author(s) and source are credited.

\section{References}

1. Gupta, V.: A note on Modified Szász operators, Bull. Inst. Math. Academia Sinica. 21(3), 275-278 (1993)

2. Gupta V., Aral A., Ozhavzali M.: Approximation by $q$-Szász-Mirakyan-Baskakov operators, communicated

3. Prasad, G., Agrawal, P.N., Kasana, H.S.: Approximation of functions on $[0, \infty]$ by a new sequence of modified Szász operators, Math. Forum 6(2), 1-11 (1983)

4. Sinha, J., Singh, V.K.: Rate of convergence on the mixed summation integral type operators. Gen. Math. 14(4), 29-36 (2006)

5. Zeng, X.M.: On the rate of convergence of the generalized Szász type operators for functions of bounded variation, J. Math. Anal. Appl. 226, 309-325 (1998) 
6. Zeng, X.M., Tao, W.: Rate of convergence of the integral type Lupas Bezier operators. J. Kyungpook Math. 43, 593-604 (2003)

7. Zeng, X.M., Cheng, X.: Pointwise approximation by the modified Szász Mirakyan operators. J. Comp. Anal. Appl. 9(4), 421-430 (2007) 